\title{
Selecting Suitable Candidates for Predictive Maintenance
}

\author{
W.W. Tiddens ${ }^{1,2}$, A.J.J. Braaksma ${ }^{1}$, and T. Tinga ${ }^{1,2}$ \\ ${ }^{1}$ University of Twente, Enschede, The Netherlands \\ w.w.tiddens@utwente.nl \\ a.j.j.braaksma@utwente.nl \\ t.tinga@utwente.nl \\ ${ }^{2}$ Netherlands Defence Academy, Den Helder, The Netherlands \\ WW.Tiddens@mindef.nl \\ T.Tinga@mindef.nl
}

\begin{abstract}
Predictive maintenance (PdM) or Prognostics and Health Management (PHM) assists in better predicting the future state of physical assets and making timely and betterinformed maintenance decisions. Many companies nowadays desire the implementation of such an advanced maintenance policy. However, the first step in any implementation of PdM is identifying the most suitable candidates (i.e. systems, components). This is to assess where PdM would provide the greatest benefit in performance and costs of downtime. Although multiple selection methods are available, these methods do not always lead to the most suitable candidates for PdM. The main reason is that these methods mainly focus on critical components without considering the clustering of maintenance, and the technical, economic, and organizational feasibility.

This paper proposes a three-stage funnel-based selection method to enhance this process. The first step of the funnel helps to significantly reduce the number of suitable systems or components by a traditional filtering on failure frequency and impact on the firm. In the second and third step, a more in-depth analysis on the remaining candidates is conducted. These steps help to filter potential showstoppers and study the technical and economic feasibility of developing a specific PdM approach for the selected candidates. Finally, the proposed method is successfully demonstrated using two distinct cases: a vessel propulsion system and a canal lock.
\end{abstract}

\section{INTRODUCTION}

Preventing unexpected failures from occurring is important for many complex systems such as production systems, medical equipment and high-tech products. Executives in such asset-intensive industries often regard unexpected failures of their physical assets as the primary operational risk to their business (LaRiviere, McAfee, Rao, Narayanan, \&

\footnotetext{
Wieger Tiddens et al. This is an open-access article distributed under the terms of the Creative Commons Attribution 3.0 United States License, which permits unrestricted use, distribution, and reproduction in any medium, provided the original author and source are credited.
}

Sun, 2016). Such unexpected downtime can be disruptive in complex manufacturing supply chains and imposes high costs due to forgone productivity (LaRiviere et al., 2016). Competitive pressure therefore forces companies to use the reliability and dependability of their equipment as a competitive weapon (Simões, Gomes, \& Yasin, 2016).

Typically, a lot of preventive maintenance is conducted to avoid negative impacts (such as safety hazards, production losses, logistic costs, or high repair costs) caused by failures. Ideally, predictive maintenance strategies are employed to provide insight in the future state of assets. Predictive maintenance (PdM) techniques inform the asset owner or operator about the current and preferably also future state of their assets. PdM thereby helps to reduce unexpected failures, improve the reliability and dependability of equipment and prevent unnecessary replacement of components. Next to that, system level monitoring can be used to get control and system performance data.

Predictive maintenance is enabled by PHM (prognostics and health management) technologies in response to the indicated deteriorated condition, performance or the remaining useful life (RUL) of a component or system (Lei \& Sandborn, 2016). Although predictive maintenance is often referred to as CBM (condition-based maintenance), predictive maintenance goes further than CBM by also taking prognostic information into account (Shafiee, 2015; Tinga \& Loendersloot, 2014).

Typically, two motivations for implementing PdM can be discerned. Either (1) industrial practitioners look for the best maintenance approach (also termed: maintenance policy selection) for their high-impact components and systems, and PdM appears to be the most suitable approach. Or (2) opportunities arise that make the application of PdM feasible, for example by digitization of assets (an example of this will be shown in section 5.2: the canal lock case).

By having the desire to implement a PdM approach, by either of these two motivations, the first step is to consider whether PdM is indeed the most suitable approach (for candidates from the first perspective) or to identify the most suitable 
candidates (i.e. systems or components) for PdM (for candidates following the second perspective) (Lee et al., 2014).

The purpose of selecting the most suitable candidates for PdM is to assess where PdM would provide the greatest benefit in terms of performance and/or cost of downtime (Lee et al., 2014). Since many maintenance techniques that enable PdM are costly to develop, it would not be cost-efficient to apply them on all equipment (Bengtsson \& Jackson, 2004). That is to say, almost $30 \%$ of industrial equipment does not benefit from applying techniques (i.e. condition monitoring) that enable CBM (or similarly PdM / PHM) (Hashemian \& Bean, 2011). It is therefore often stated that CBM should only be applied where it is suitable, not as an overall policy (Moubray, 1997). It is therefore critical to select the suitable candidates for a PdM application to achieve the optimal benefits (Brahimi, Medjaher, Leouatni, \& Zerhouni, 2016).

Therefore, in this paper, a method is developed to identify and select suitable candidates for predictive maintenance. A design science method (Holmström, Ketokivi, \& Hameri, 2009) is followed in developing the selection method. First, section 2 gives an overview of current methods. After this, section 3 covers the problem exploration (step 1). The problem exploration serves as design criteria for the initial solutions (step 2), which are proposed in the literature review and in the reflection of existing methods. The solution is designed (step 3) in section 4 to identify suitable candidates from the plethora of components that are typically available in complex systems. The solution is demonstrated (step 4) in section 5 in two distinct cases: a propulsion system of a ship and a canal lock. Finally, concluding remarks will be given in section 6.

\section{REVIEW OF CURRENT METHODS}

The literature describes various well-known and accepted methods to select suitable candidates for PdM. In this section, we will first discuss various methods that have been proposed to identify or select critical components. In section 3 , we will discuss the shortcomings of current methods and how the discussed methods can contribute to an improved approach to select the suitable candidates for PdM.

\subsection{Methods based on risk assessment and dependability of components}

An often applied approach is to define critical components as a component whose failure leads to unavailability of the whole system, and/or a component which has a high failure rate (Gouriveau, Medjaher, \& Zerhouni, 2016).

A first alternative for this is to define the critical components of a system by conducting a dependability analysis (Brahimi et al., 2016). A dependability analysis brings components to light whose failures have the highest impact on the availability, reliability, maintainability, safety, and integrity of the system (Avizienis, Laprie, Randell, \& Landwehr, 2004). Qualitative dependability analyses use expert judgment to evaluate potential failures to evaluate risk, using for example a Failure Mode Effects (and Criticality) Analysis (FMEA and FMECA) (Brahimi et al., 2016) or a Fault Tree Analysis (FTA). Quantitative dependability analyses use statistical methods and can be deterministic or probabilistic. These methods are used to estimate measures such as the mean time to failure (MTTF), mean time between failures (MTBF), or failure rates to evaluate a system's reliability (Brahimi et al., 2016).

A quantitative approach that follows this logic is to select the top 10 cost drivers or availability killers for a PdM policy, as done in for example the degrader analysis of Banks, Reichard, Hines, and Brought (2008). This approach has similarities with an often applied Pareto analysis based on maintenance costs, failures, downtime, or safety.

The reliability-centered maintenance (RCM) method (Moubray, 1997) uses systematic logic to rank the criticality of failure modes and provides guidelines for selecting the applicable maintenance task (Tsang, 1995). RCM is normally performed at the system level since the criticality of failures at the component level can only be judged on the basis of its impact on delivering the required system functions (Tsang, 1995).

An example of such a dependability analysis that uses RCM logic is the Most Important Systems (MIS) and Most Critical Components (MCC) analysis of Waeyenbergh and Pintelon (2002). Their analysis first focuses on selecting the so called most important systems by regarding the impact of its breakdown on safety, environment, production, repair costs, and secondary damages. This includes bearing in mind the system's ease of repair and the ease of failure detection. Next to that, in the MIS analysis it is considered whether the system is a bottleneck, redundant, or complex. The second step, the MCC analysis, helps to determine the most critical components within the selected system (the MIS). This is done with a simplified FMECA, which is according to the authors "rather 'quick and dirty', but [...] very easy to use". In this simplified analysis, the same consequences are considered as is done in the MIS analysis. RCM cannot only be used to select the most critical components, based on for example their risk priority number (RPN), but also advises on the most suitable maintenance task.

Dehghanian, Fotuhi-Firuzabad, Bagheri-Shouraki, and Kazemi (2012) proposed a fuzzy-AHP (analytical hierarchy process) method to identify critical components in a RCM program. They prioritize components based on: (i) the number of failures, (ii) the number of component failures, (iii) repair duration, (iv) component investment costs, and (v) component repair and maintenance costs.

Lee, Liao, Lapira, Ni, and Li (2009) argue that identifying the critical components on which the PdM approach should be 
performed is the first key step by deciding which components' degradation has significant impact to the system's performance or costs a lot when the downtime happens. They propose to use a four-quadrant chart which quickly distinguishes between components based on their failure rate (low vs high) and their associated downtime (low vs high). Lee et al. (2014) argue that predictive maintenance should only be applied on those components that have a critical impact on the firm (i.e. high downtime) and a low failure rate. Note that components with a high failure rate and high associated downtime should be designed out.

Labib (2004) proposed a similar idea that helps to determine the suitable maintenance policy for components based on a trade-off between the frequency of failure and the downtime. In their approach, a condition-based concept is only applied to those components that have a low frequency of failure but a high associated downtime. This is comparable to the approach of Lee et al. (2009).

\subsection{Maintenance policy selection methods}

Maintenance policy selection methods do not directly help to select the most suitable candidate for predictive maintenance. However, as was also mentioned in the introduction, the starting point for many companies is to find a suitable policy for their high-impact components and systems. When PdM is selected to be most appropriate, a check on the suitability of the candidates might prove valuable. Moreover, reviewing these policy selection methods may provide valuable insights into when to select PdM for a (component in the) system as it provides rules or criteria for when to apply predictive maintenance strategies.

Different goals and criteria can be taken into account in the selection of the most suitable maintenance policy. A maintenance policy can be defined as a rule or set of rules that describe the triggering mechanism, such as time, usage or an expression of deterioration, for the different maintenance actions (repair, replace, monitor, shutdown) (Pintelon \& Van Puyvelde, 2006). To take these various criteria and goals into account, multi-criteria decision making (MCDM) methods can be used for maintenance policy selection (MPS). The MCDM approach is typically suitable in contexts where the goals and criteria are difficult to express in monetary terms and are therefore difficult to quantify. For MPS, these criteria include the investment required for implementation, safety aspects, environmental issues, failure costs, reliability of the strategy, and manpower utilization of the facility (Shafiee, 2015).

Shafiee (2015) reviewed the use of MCDM methods for MPS. MPS, which is by other authors sometimes also termed maintenance strategy selection (MSS), helps practitioners in selecting between policies such as corrective maintenance (CM), preventive maintenance (PM), opportunity-based maintenance or predictive maintenance. Goossens and Basten (2015) proposed a maintenance policy selection method for naval ships based on a multi-criteria decision making (MCDM) method that helps to select between failurebased maintenance, time or use-based maintenance, and condition-based maintenance. In their method they consider whether the maintenance policy fits the: (i) crew's size and education level, (ii) available knowledge in the company, (iii) mission profile, (iv) internal and external relations of the company, and finally (vi) influences on performing maintenance tasks.

\section{Problem EXPloration: SHORTCOMINGS OF EXISTING METHODS}

Based on practical experience and literature, the problem exploration in this subsection will point out several factors that cause that traditional selection methods not always lead to the best selection of components.

\subsection{Underestimating time consuming process}

Even though a PdM policy might be the preferable strategy for an asset, not all components within that asset might be suitable for PdM. FMECA analyses can be used to identify suitable components. However, FMECAs can become very extensive and time-consuming when they are conducted down to the component level for a complex system.

Tinga, Tiddens, Amoiralis, and Politis (2017) for example, showed that a complex asset like a ship typically can be decomposed in up to 60 installations. In case of a naval vessel, the installations within the propulsion section can be formulated on the level of a gearbox, thruster or diesel engine. But these installations again contain numerous components, e.g. main bearing, connecting rod, cylinders and pistons for the diesel engine.

Approaches such as streamlined FMECA or simplified FMECA (as used in the approach of Waeyenbergh and Pintelon (2002)) can help to reduce this complexity by first focusing on the criticality of the installation / subsystem. However, these approaches do not guarantee that all critical components have been identified (Tinga et al., 2017). Therefore, methods like a recursive combination of FTA and FMEA have been proposed (Peeters, Basten, \& Tinga, 2018). In this method, FMEA is used to assess the criticality of system level failure modes that are identified in a FTA. For the selected (critical) failure modes, a function level FTA is conducted followed by another FMEA.

Since applying traditional methods can lead to a time consuming process, it would be useful to find ways that can reduce the required effort.

\subsection{Ignoring clustering of maintenance activities}

Only being a critical component does not make a component a suitable component for PdM. Using prognostic methods to extend the component's lifetime is only useful when the failure prediction actually enables reducing or extending the 
maintenance intervals. Interval reductions typically mean that failures that otherwise would have occurred can be prevented, thereby leading to an increase in the system availability. Interval extensions lead to cost reductions (conducting less preventive maintenance) and thereby also higher availability of the asset (as it is not in maintenance).

Maintenance activities however, are often clustered based on a production (i.e. opportunistic maintenance planning in a production plant) or mission (i.e. docking intervals for a ship) planning. Such a planning dictates when maintenance activities can be conducted and thereby restrict shifting maintenance activities, unless these can be extended to the next planned opportunity. In these cases, preventive (opportunity-based) maintenance is sometimes more convenient than more advanced methods.

Clustering of maintenance activities can also be caused by technical restrictions. Take the example of gearbox maintenance. Extending the maintenance interval of a gearwheel in a gearbox would make little sense if all other parts in the gearbox have to be maintained anyway (unless it concerns a very expensive component). The benefits of extending maintenance of the gearwheel can only be achieved when all maintenance activities for the gearbox can be extended. Finally, a single maintenance activity can also contribute to preventing multiple failure modes from occurring. This means that it might be possible to extend a maintenance activity by monitoring a specific failure mode, but that another failure mode can become dominant when this maintenance activity is extended.

\subsection{No consideration of technical feasibility}

Although an imminent failure might be beneficial to monitor, it is not always possible to predict or detect the anomaly before a failure occurs. Besides, prognostics can never be $100 \%$ sure to predict failures and faults (Jardine, Lin, \& Banjevic, 2006). Moreover, analyzing data sets without knowing the underlying failure mechanisms can lead to incorrect results. This means that a root cause analysis is essential in achieving an effective maintenance policy. The technical feasibility goes hand in hand with the knowledge level within the company. Understanding of the degradation mechanism is often required to predict or detect the failure. Either internal or access to external knowledge sources is required to gain this understanding. When this is not available, the PdM approach cannot be implemented successfully. Next to this, considering the technical feasibility of PdM is important for: (i) failures due to human error (which can typically not be predicted or monitored); and (ii) failures of which the faulty condition cannot be detected early enough (early warning) in comparison to the maintenance opportunity (suppose a large part aboard a ship). The latter can cause that the prognostic model is ineffective. Finally, firms should consider whether a prognostic model is already available or that additional research has to be conducted.

\subsection{No consideration of economic feasibility}

Although costs are often mentioned as a criterion for maintenance policy selection, there are more reasons that can hinder the full potential of PdM. The four quadrant chart of Lee et al. (2009) and the selection method of Labib (2004) consider a trade-off between the failure frequency and an failure effect criterion such as downtime, costs or safety. However, these methods do not consider a lower boundary for the failure frequency or an upper boundary for the failure effect. The four quadrant method of Lee et al. (2009) was therefore first improved by Tiddens, Braaksma, and Tinga (2017) who introduced upper boundaries for the failure effect and upper boundaries for the failure frequencies; both to advise redesign as the consequences of these types of failures can disrupt firms. After that, Tinga et al. (2017) introduced lower boundaries for the failure frequency to guarantee the economic justification for PdM policies; the investment in $\mathrm{PdM}$ is not recovered when there are not 'enough' failures or these are not experienced within the realistic (economic) lifetime of the asset.

Next, the business case for predictive maintenance could be negative because: (i) introducing PdM is more costly than preventive or corrective maintenance; (ii) there is a low probability of unnecessary replacements (high confidence that failure will occur as predicted in preventive policy); (iii) probability of correct prediction of time to failure is not important as part will be replaced anyhow before each mission.

\subsection{No consideration of organizational feasibility}

Even though PdM might be economically and technically feasible, it should also fit the organization. Issues such as a lack of system and domain knowledge, a lack of trust in monitoring systems and the organization not being ready to implement PdM can hinder the adoption of PdM. Jonsson and Westergren (2004) clearly expressed the opinion of one employee towards the adoption of PdM: "What will break first - the motor or the remote [monitoring] system?”. Jonsson and Westergren (2004) argue that firms should work hard on showing the added value of the system or on integrating it with a larger monitoring system to overcome this barrier.

\subsection{No focus on desired use and desired outcome of predictive maintenance approach}

The reviewed methodologies pay little attention to the different possible outcomes of the selected predictive maintenance approach. In the literature, three types of end results of a prognostic approach are often distinguished: detection, diagnosis, and prognosis (Jardine et al., 2006). The inputs from a condition monitoring (CM) system can be used 
for CBM, but also (in a simplified manner) to only detect anomalies (detection). These differences in outcomes should be included in the selection of suitable candidates as this difference leads to another view on the discussed limitations of existing methods.

For example, considering the clustering of maintenance activities (as discussed in 3.2) is not important for detecting anomalies (for example preventing safety incidents) while it is important for extending maintenance actions (the goal of prognosis). Although PdM/PHM and CBM are sometimes used interchangeable, they lead to different end results. CBM is a dynamic maintenance policy based on performance and/or condition monitoring. CBM thereby aims to detect anomalies in the operation of industrial machinery: the discovery of changes in their characteristics prefigures a future failure in the short term (Gouriveau et al., 2016). Where CBM is mainly diagnostic, PHM (used for PdM) includes prognostic capabilities (Tinga \& Loendersloot, 2014). We therefore consider CBM as diagnosis and PdM / PHM as prognosis. Detection (using e.g. CM) is typically used to recognize imminent failures before the system fails and can therefore be seen as a safety warning.

\subsection{Factors to consider}

The factors discussed before in this section serve as design criteria for the final solution development, that should help to reduce time consumption of the process (following section 3.1). These factors have been added to the already known factors to create the list shown in Table 1, now containing all factors that can hinder the usage of predictive maintenance. The overview of Goossens and Basten (2015) was used as starting point, and this list was extended with the factors discussed in sections 3.1 to 3.6 (marked with '*').

\begin{tabular}{|l|}
\hline Factors hindering the use of PdM \\
\hline Clustering \\
\hline No consideration of production/mission clustering * \\
\hline No consideration of technical clustering \\
\hline Technical feasibility \\
\hline Other failure modes become critical $*$ \\
\hline Failure cannot be predicted due to human errors * \\
\hline Failure cannot be detected with existing technology * \\
\hline Failure cannot be detected even with additional research * \\
\hline Failure cannot be predicted with existing technology \\
\hline Failure cannot be predicted even with additional research $*$ \\
\hline Economic feasibility \\
\hline Insufficient financial resources available * \\
\hline Not enough failures (during life time) \\
\hline Organizational feasibility \\
\hline No trust in monitoring system \\
\hline No fit to crew (size and education level) \\
\hline No fit to existing knowledge levels \\
\hline No fit to the operational task (maintenance location) \\
\hline No fit to mission (equipment age, location and profile) \\
\hline
\end{tabular}

Not compliant with existing policies and prescriptions

No fit to (external) relations (outsourcing)

No fit to the spare parts (commonality, availability)

Table 1. Factors that hinder the use of predictive maintenance (new factors are marked with '*')

\section{SOLUTION DEVELOPMENT: PROPOSED SOLUTION FOR IDENTIFICATION OF SUITABLE CANDIDATES FOR PDM}

\subsection{Proposed method}

The proposed methodology (visualized in Figure 1) consists of three stages: the criticality classification, the identification of showstoppers, and a focused feasibility study. The proposed method works as a funnel, the first stage aims to reduce the number of potential candidates significantly to reduce the required efforts in the two following stages. Each of the three stages will be elaborated in the following subsections, and demonstration of the proposed method in two real cases then follows in section 5 .

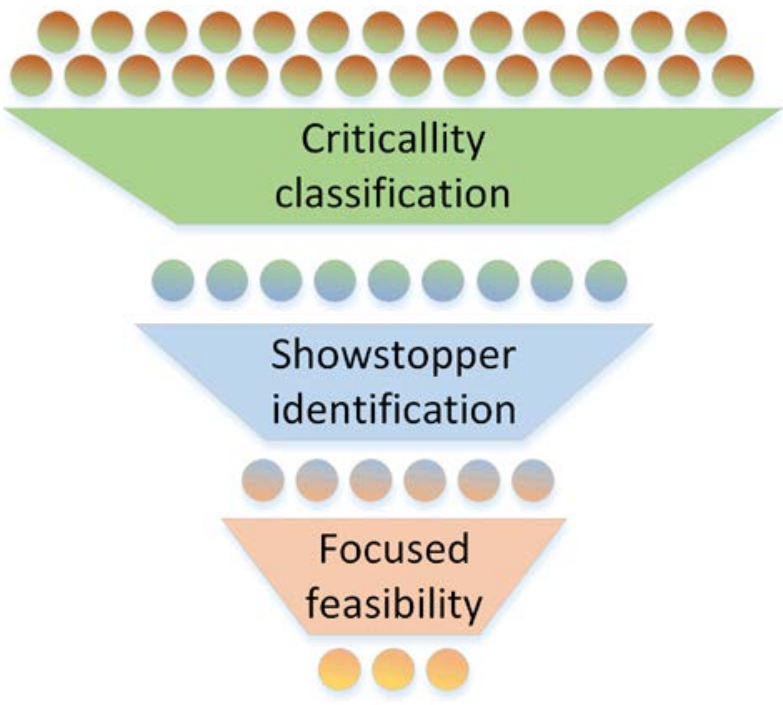

Figure 1. Proposed funnel approach for the identification of suitable candidates for PdM

\subsection{Criticality classification}

The initial criticality classification acts as a filter to significantly reduce the number of possible candidates from a plethora of components. The four quadrant chart based on the work of Labib (2004), Lee et al. (2009) and Tinga et al. (2017) helps to bring focus to only the most promising candidates, namely those with a low frequency of failure and a high associated failure consequence (e.g. failure, costs or downtime). The improved focus established by the introduction of a lower limit (in addition to the upper limit) on the failure frequency helps to only select those candidates that fail often enough for a positive business case. 


\subsection{Identifying showstoppers related to the desired outcome}

Showstoppers are factors that can make the prognostic approach infeasible or providing no added value. In section 3 several factors were identified that hinder the use of PdM / PHM. These are clustered in the potential showstoppers (PS) listed in Table 2.

Firstly, following section 3.6, a differentiation is made between three ambition levels, the desired results of prognosis: Detection: used as safety warning or last resort; Diagnosis: determine fault state and short-term (failure) behavior forecast; and Prognosis: long-term (failure) behavior prediction. Determining the desired outcome by differentiating between detection, diagnosis, and prognosis helps to firstly describe the requirements of the prognostic system and secondly explore the possibilities and impossibilities by recognizing the potential showstoppers. Next, when considering technical feasibility, a choice has to be made whether additional research may be conducted or only existing technologies can be applied.

Per approach, it is then determined whether the potential showstopper is present in the situation under analysis, which then prevents that approach to be successful. For practitioners, it will however not always be trivial whether a factor will be a showstopper. Three possible outcomes can therefore be chosen: Yes (it is a showstopper), No (it is not a showstopper), or Maybe (it might be a showstopper). In the final stage of the procedure, the focused feasibility study (section 4.4), these Maybe's will be looked at in more detail. When no other showstoppers have already made the desired approach infeasible, the Maybe has to be transformed in a decisive Yes or No in this final stage.

The potential showstoppers (listed in Table 2) can be operationalized in the following manner:

Mission clustering: can a possible PdM activity be conducted during or in-between missions? If not, can the predicted maintenance activity be planned with a minimum of the duration of one operational period in advance (i.e. this requires a prognostic distance of at least one mission duration)?

Technical clustering: does the predicted failure mode drive the package of clustered maintenance activities (i.e. does it drive the maintenance interval)? If not, can it be skipped one interval? Note: this also requires considering whether all relevant failure modes of the component are listed.

Detecting/predicting with existing/additional research: is it viable to build a model that is able to detect/predict the failure mode/mechanism with existing/additional research?

Sufficient financial resources: are sufficient resources available to cover the investment costs?
Enough failures during life time: will sufficient failures occur (and thus be prevented under a predictive maintenance policy) to recover the investment costs? Note: the lower limit line in the four quadrant chart also helps to cover this PS.

Trust in monitoring system: will the developed monitoring system be trusted by maintenance personnel and operators?

Fit to personnel: is sufficient knowledge, qualifications and experience with maintenance available within the company? Is there sufficient willingness to adopt PdM?

Operational task and mission: does the predictive maintenance fit with performing the operational mission?

Relations and policies: does the predictive maintenance fit with the internal and external relations of the company?

Spare parts: does the predictive maintenance policy fit with the type, commonality and availability of spares?

\begin{tabular}{|c|c|c|c|c|}
\hline & Potential Showstoppers (PS) & 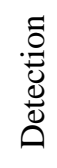 & 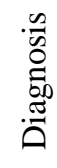 & $\begin{array}{l}. \mathscr{n} \\
\tilde{0} \\
0 \\
0 \\
0 \\
0\end{array}$ \\
\hline \multicolumn{5}{|c|}{ Clustering } \\
\hline c1 & $\begin{array}{l}\text { No match with production or } \\
\text { mission planning }\end{array}$ & & PS & PS \\
\hline $\mathrm{c} 2$ & No match with technical clustering & & PS & PS \\
\hline \multicolumn{5}{|c|}{ Technical feasibility } \\
\hline t1a & $\begin{array}{l}\text { Failure cannot be detected with } \\
\text { existing technology }\end{array}$ & PS & PS & \\
\hline $\mathrm{t} 1 \mathrm{~b}$ & $\begin{array}{l}\text { Failure cannot be predicted with } \\
\text { existing technology }\end{array}$ & & & PS \\
\hline $\mathrm{t} 2 \mathrm{a}$ & $\begin{array}{l}\text { Failure cannot be detected with } \\
\text { additional research }\end{array}$ & PS & PS & \\
\hline $\mathrm{t} 2 \mathrm{~b}$ & $\begin{array}{l}\text { Failure cannot be predicted with } \\
\text { additional research }\end{array}$ & & & PS \\
\hline \multicolumn{5}{|c|}{ Economic feasibility } \\
\hline e1 & Insufficient financial resources & PS & PS & PS \\
\hline e2 & $\begin{array}{l}\text { Not enough failures (during life } \\
\text { time) for positive business case }\end{array}$ & PS & PS & PS \\
\hline \multicolumn{5}{|c|}{ Organizational feasibility } \\
\hline 01 & No trust in monitoring system & PS & PS & PS \\
\hline 02 & No fit to personnel & PS & PS & PS \\
\hline 03 & No fit to operational task / mission & & PS & PS \\
\hline 04 & No fit to relations and policies & & PS & PS \\
\hline 05 & No fit to the spare parts & & PS & PS \\
\hline
\end{tabular}

Table 2. Identification of potential showstoppers (PS) for the differentiated application of PdM

Table 2 shows that each of the 11 factors can act as a showstopper for the two most ambitious (Diagnosis and Prognosis) levels, but only six of them could affect the lower level Detection.

Showstoppers that are identified on the aspects "organizational feasibility" and "clustering" can result in a 
(temporary) stop on developing the desired PdM approach. Therefore, analysis is required to see how these showstoppers can be mitigated. For the clustering, it should be examined how the candidate can be removed from the maintenance (either mission or technical) cluster or whether the interval of the other maintenance activities within the cluster can be coupled to the selected candidate.

For the organizational feasibility, the identified showstoppers point at factors that have to be addressed before PdM can be successfully implemented. Bengtsson (2008) provides a checklist with organizational factors to take into consideration when implementing PdM. Bengtsson argues that when a positive business case can be made for the implementation, small scale pilot projects and gradual implementation can help to overcome these barriers.

The showstoppers on the aspects "technical feasibility" and "economic feasibility" can also result in a (temporary) stop on developing the desired PdM. However, for these factors the answer is not always a clear Yes or No, so these will be addressed in the third and final stage, the focused feasibility study.

\subsection{Focused feasibility study}

In the final stage of the method, the feasibility of developing a prognostic model is further examined for those candidates where a 'Maybe' has been selected for one or several of the showstoppers (and no definite showstoppers were identified). This means that these factors will be studied in more detail.

The economic feasibility study focuses on whether developing the prognostic model is beneficial to the firm, from a strategic point of view. This is because not all industrial equipment benefits from the application of prognostic techniques. It is difficult to assess the financial impact with a high accuracy at the start of the project, but it is important to discuss and brainstorm on the possible gains in comparison to the possible investment costs (Bengtsson, 2008). As proposed by Tiddens, Brouwer, Braaksma, and Tinga (2017), first a distinction should therefore be made between explorations and exploitations of PdM / PHM. Exploitations regard applying well-known (to the firm) technologies. For these, a detailed financial modelling can be executed, using for example the method proposed by Tiddens, Brouwer, et al. (2017). This method helps to determine whether developing PdM for the candidate system leads to a financial justification. In these cases, it is often quite clear whether the use of PdM is beneficial to the firm and the showstopper identification can be conducted with limited uncertainties. Explorations are those developments in which the frontiers of what is known (within the firm) are pushed. For explorations, a detailed cost benefit analysis cannot be made since the costs and benefits are highly uncertain. Then, a maintenance balanced scorecard approach can be used. An example of this approach, proposed by Alsyouf (2006), is shown in Table 3. A multi-criteria decision making approach could in this situation be used to compare $\mathrm{PdM}$ with fixed-interval preventive maintenance (PM) and corrective maintenance (CM) (or any other maintenance policy) at a strategic level. Although PdM was - technology wise - possibly already selected as the preferable maintenance approach for the candidate system, the highest total score (22) for PdM in Table 3 confirms that in this case developing PdM is of strategic interest to the firm.

\begin{tabular}{lccc}
\hline Perspective & CM & PM & PdM \\
\hline (i) innovation and growth & 1 & 2 & 4 \\
\hline (ii) maintenance & 2 & 3 & 3 \\
\hline (iii) production & 1 & 3 & 3 \\
\hline (iv) customer & 1 & 4 & 5 \\
\hline (v) society & 1 & 3 & 4 \\
\hline (vi) financial & 2 & 3 & 3 \\
\hline Total & $\mathbf{8}$ & $\mathbf{1 8}$ & $\mathbf{2 2}$ \\
\hline
\end{tabular}

Table 3. Example multi-criteria analysis to assess the (positive) impact of PdM (1 = low, 5 = high)

The technical feasibility study focuses on whether it is possible for the firm to develop and implement the desired prognostic approach. The technical feasibility goes deeper than the showstopper identification. Determining the feasibility is not always trivial, since for innovative PdM approaches, a successful outcome can be quite uncertain. In the technical feasibility study, focus should be paid to the seven functional levels that are used in the OSA-CBM (Open System Architecture for CBM) (Lebold, Reichard, \& Boylan, 2003). The level of detail of this technical feasibility study should be determined per case. In case of a highly uncertain and costly PdM implementation a complete proof-of-concept might be required, whereas in case of a low cost and low risk application, only briefly discussing the questions would be sufficient. Following the requirements defined by Gouriveau et al. (2016), based on the original OSA-CBM, the following questions guide the assessment of the technical feasibility:

1. Data acquisition: How can the failure mechanism be measured? How can the required data be acquired, backupped and secured?

2. Data processing: How can the signals issued from the sensors be processed to extract features that suggest the presence of anomalies, and in the long term, represent the state of the monitored system?

3. Detection (condition assessment): How can the real-time data be compared with expected or known values. How can alerts be generated based on criteria of performance, security, etc.?

4. Diagnostics (not required for: 'Detection'): How can based on the detected state - be determined whether the monitored system is degraded? How can insight be provided on influences of interactions with other components, operating and environmental conditions?

5. Prognostics (not required for: 'Detection' and 'Diagnosis'): How can the current state of the monitored 
system be determined? How can the future state of the monitored system be determined and an estimation of the remaining useful life (RUL) be given?

6. Decision analysis: How can maintenance/control actions be recommended such that the system can function until the accomplishment of the mission?

7. Presentation: How can the results be presented?

\section{SOLUTION DEMONSTRATION}

\subsection{Selecting suitable candidates for PdM for a ship's propulsion system}

MAR is a maritime company that monitors the seas and collects engineering and earth data. For its vessels, MAR wants to introduce PdM and has therefore joined the MaSeLMA (maintenance and service logistics for maritime assets) research project. In this project (in which the authors are also involved), MAR cooperates with other maritime companies to develop a PdM approach for the diesel-direct propulsion system of a series of their vessels. The first step for MAR therefore is determining the most suitable candidates for PdM within the propulsion system. As multiple companies are involved in this project, the final selection is based on the result of the identification of suitable components at multiple companies. For clarity, this case study only discusses the selection of suitable candidates at MAR.

\subsubsection{Criticality classification}

A streamlined FMECA has been conducted to determine the most critical failure modes within the diesel-direct propulsion system of MAR. These FMECAs were conducted using experts at MAR and external experts from the supplier of the diesel engines. The identified failures are plotted in the four quadrant chart, see Figure 2. The four quadrants represent the following maintenance advice: Q1 (upper right, including the cut-off regions of Q2 and Q4): fix unreliability during design; Q2 (upper left): fix failures with spare parts; Q3 (lower left): regular (OEM prescribed) maintenance; and Q4 (lower right): predictive maintenance. The dotted line indicates the lower limit for Q4, failures with a lower failure frequency are expected to lead to a negative business case. Eleven failures (Table 4) are identified in the Q4 area. These failures cause a downtime longer than 24 hours and have a failure occurrence of more than once every 30 years (the minimum economic lifetime of the vessel). The upper limit on the failure frequency for Q4 is set at one failure every three years.

\subsubsection{Identifying showstoppers related to the desired outcome}

MAR has the desire to do prognostics, so the associated potential showstoppers are analyzed ( $t 1 a$ and $t 2 a$ are not applicable for prognostics). MAR operates it's vessels all over the world. Consequently, their maintenance opportunities are limited. The current fixed-interval preventive maintenance policy prescribes many clustered maintenance activities ( $c 2$ ). The larger maintenance activities are scheduled during docking periods. The mission schedule allows for small maintenance activities during operations and bad weather periods (o3). However, at those moments limited tools and specialisms are available thus not all maintenance activities can be conducted (c1).

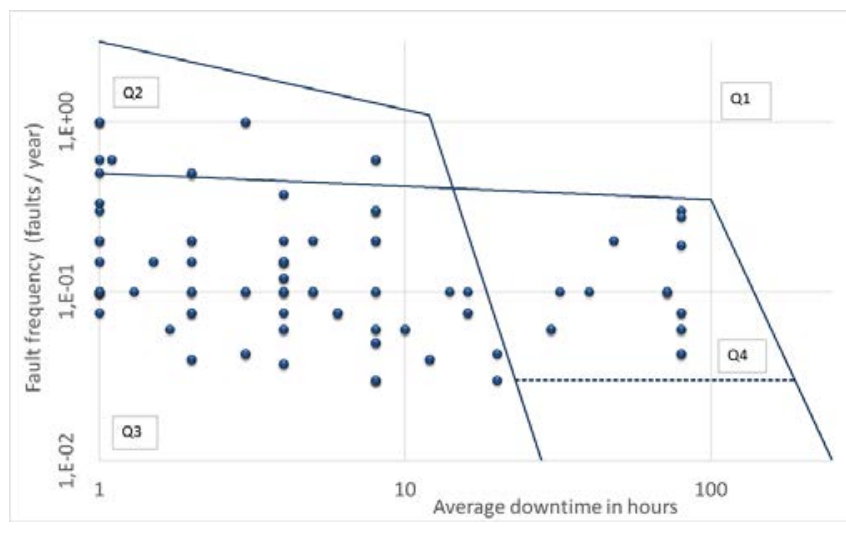

Figure 2. Four quadrant method, showing failures for MAR's vessel propulsion system.

\begin{tabular}{|l|l|}
\hline ID & Description of event / failure mode \\
\hline 1 & Diesel engine - bearing shells worn \\
\hline 2 & Diesel engine - connecting rod bearing shell worn \\
\hline 3 & $\begin{array}{l}\text { Diesel engine - pistons ( + springs) seized / fouled } \\
\text { due to liner wear }\end{array}$ \\
\hline 4 & $\begin{array}{l}\text { Diesel engine - valve mechanism - valve broken } \\
\text { due to fatigue / overload / wear }\end{array}$ \\
\hline 5 & Diesel engine - gear wheel train worn \\
\hline 6 & Diesel engine - air cooler waterside fouled \\
\hline 7 & $\begin{array}{l}\text { Gearbox - gearwheels teeth worn / broken due to } \\
\text { wear / overload }\end{array}$ \\
\hline 8 & Gearbox - thrust bearing worn \\
\hline 9 & Thruster - coupling slipped due to wear \\
\hline 10 & Thruster - frequency converter failed \\
\hline 11 & Thruster - encoder failure due to software error \\
\hline
\end{tabular}

Table 4. Identified candidates using the four quadrant chart method

Next, the different crews aboard the vessels are to be trained in the use of prognostic systems (o2). Moreover, high reliability is demanded in the industry and trust in the monitoring systems has to be gained in time (o1). Finally, limited funds are available to invest in monitoring systems. Therefore, the cost effectivity of the investments in PdM is 
key for MAR $(e 1, e 2)$. Table 5 shows the complete overview of identified showstoppers for the candidates remaining from the first step (the four quadrant chart).

\begin{tabular}{|c|c|c|c|c|c|c|c|c|c|c|c|c|}
\hline ID & 기 & $\mathcal{V}$ & $\hat{\exists}$ & จิ & ন & ชี & $\bar{o}$ & ชิ & $\ddot{\theta}$ & I & 농 & $\underset{\mathscr{\varpi}}{\mathscr{\Xi}}$ \\
\hline 1 & $\mathrm{~N}$ & $\mathrm{Y}$ & $\mathrm{M}$ & $\mathrm{N}$ & $\mathrm{N}$ & $\mathrm{N}$ & $N$ & $\mathrm{~N}$ & $\mathrm{~N}$ & $\mathrm{~N}$ & $\mathrm{~N}$ & $\mathrm{Y}$ \\
\hline 2 & $\mathrm{~N}$ & $\mathrm{Y}$ & $\mathrm{M}$ & $\mathrm{N}$ & $\mathrm{N}$ & $\mathrm{N}$ & $N$ & $\mathrm{~N}$ & $\mathrm{~N}$ & $\mathrm{~N}$ & $\mathrm{~N}$ & $\mathrm{Y}$ \\
\hline 3 & $\mathrm{~N}$ & $\mathrm{Y}$ & $\mathrm{M}$ & $\mathrm{N}$ & $\mathrm{N}$ & $\mathrm{N}$ & $\mathrm{N}$ & $\mathrm{N}$ & $\mathrm{N}$ & $\mathrm{N}$ & $\mathrm{N}$ & $\mathrm{Y}$ \\
\hline 4 & $\mathrm{~N}$ & $N$ & $\mathrm{M}$ & $\mathrm{N}$ & $\mathrm{N}$ & $\mathrm{N}$ & $\mathrm{N}$ & $\mathrm{N}$ & $\mathrm{N}$ & $\mathrm{N}$ & $\mathrm{N}$ & $\mathrm{M}$ \\
\hline 5 & $\mathrm{~N}$ & $\mathrm{M}$ & $\mathrm{N}$ & $\mathrm{N}$ & $\mathrm{N}$ & $\mathrm{N}$ & $\mathrm{N}$ & $\mathrm{N}$ & $\mathrm{N}$ & $\mathrm{N}$ & $\mathrm{N}$ & $\mathrm{M}$ \\
\hline 6 & $\mathrm{~N}$ & $\mathrm{M}$ & $\mathrm{N}$ & $\mathrm{N}$ & $\mathrm{N}$ & $\mathrm{N}$ & $\mathrm{N}$ & $\mathrm{N}$ & $\mathrm{N}$ & $\mathrm{N}$ & $\mathrm{N}$ & $\mathrm{M}$ \\
\hline 7 & $\mathrm{~N}$ & $\mathrm{~N}$ & $\mathrm{~N}$ & $\mathrm{~N}$ & $\mathrm{~N}$ & $\mathrm{~N}$ & $\mathrm{~N}$ & $\mathrm{~N}$ & $\mathrm{~N}$ & $\mathrm{~N}$ & $\mathrm{~N}$ & $N$ \\
\hline 8 & $\mathrm{~N}$ & $N$ & $\mathrm{M}$ & $\mathrm{N}$ & $\mathrm{N}$ & $\mathrm{N}$ & $\mathrm{N}$ & $\mathrm{N}$ & $\mathrm{N}$ & $\mathrm{N}$ & $\mathrm{N}$ & $\mathrm{M}$ \\
\hline 9 & $\mathrm{~N}$ & $N$ & $\mathrm{~N}$ & $N$ & $\mathrm{~N}$ & $\mathrm{~N}$ & $N$ & $N$ & $\mathrm{~N}$ & $\mathrm{~N}$ & $N$ & $N$ \\
\hline 10 & $\mathrm{~N}$ & $\mathrm{~N}$ & $\mathrm{M}$ & $N$ & $\mathrm{~N}$ & $\mathrm{~N}$ & $\mathrm{~N}$ & $\mathrm{~N}$ & $\mathrm{~N}$ & $\mathrm{~N}$ & $\mathrm{~N}$ & $\mathrm{M}$ \\
\hline 11 & $\mathrm{~N}$ & $N$ & $\mathrm{Y}$ & $\mathrm{M}$ & $\mathrm{N}$ & $\mathrm{N}$ & $Y$ & $\mathrm{~N}$ & $\mathrm{~N}$ & $\mathrm{~N}$ & $N$ & $Y$ \\
\hline
\end{tabular}

Table 5. Identification of showstoppers for the 9 candidates (ID) at MAR. $\mathrm{Y}=$ yes, $\mathrm{N}=$ no, $\mathrm{M}=$ maybe.

\subsubsection{Focused feasibility study}

The showstopper identification shows that the candidates with ID:4,5,6,8,10 are 'Maybe' suitable for PdM. The technical feasibility will therefore be studied in more detail in this section, focusing on the valve mechanism (ID:4). Studying the economic feasibility in detail is not necessary as developing and applying a PdM approach is expected to result in financial savings. The technical feasibility of developing PdM for the valve mechanism has been studied by Duplex (2017), the results are presented here along the seven levels of the OSA-CBM structure.

1. Data acquisition: based on the work conducted by Lewis, Dwyer-Joyce, Slatter, and Brooks (2004), a physical model has been developed. This model accounts for impact (when the valve closes) and abrasion (sliding of valve and seat under combustion pressure). The former is estimated by an empirical relation used in erosion studies, and the latter by Archard's wear law. These are then summed to make a final wear prediction. Key input parameters for the model are thermodynamic working characteristics of the engine, material properties, and engine operational scenarios. The required data will be collected from engine operational parameters, simulations, experiments and empirical values from literature. The operational profile will be estimated in discussions with ship managers. If the results are promising then a data acquisition system will be installed to record actual operational hours and load conditions.

2. Data processing: based on the estimated or logged operational profile, a wear rate can be determined using the developed physical model.

3./4. Detection and Diagnostics: The model output will be compared with manufacturer instructions for rejection criteria. The actual wear profile of valves can be determined for a given or logged set of operational scenarios.

5. Prognostics: Based on the estimated future operational scenario, the model calculates a remaining useful life (RUL).

6./7. Decision analysis and Presentation: The decision support tool will be customized to determine actual wear and remaining life of valves. Subsequently, a set of working scenarios can be simulated in this tool to estimate an approximate maintenance interval or assist in mission planning.

\subsubsection{Discussion on MAR case}

The showstopper identification proves to be of high value for the MAR case. Initially, the cylinder liners (ID:3) were selected in the MaSeLMA project. This selection was made by conducting the FMECA analyses and applying the four quadrant chart only. During the project it however turned out that the developed physical model, that helped to prolong maintenance intervals, could not be used to its optimum. As the maintenance activities of the complete diesel engine are clustered into a use-based preventive maintenance policy, only conducting maintenance for the liners would not be efficient. The showstopper identification, as used in the proposed method, clearly points at this issue (c2). By using the proposed method, the clustering of maintenance activities could have been identified in advance as a showstopper.

Further, one of the factors affecting the failure: diesel engine bearing shells worn (ID:1) is fouling of lubrication oil. Periodic oil sample analysis is already ongoing at MAR. However, considering the mission clustering (c1) requires checking whether the prognostic distance of these analyses is large enough. Oil samples cannot be analyzed aboard all vessels. These samples are therefore send to a lab. However, when the vessel is operating in a remote location, the period between taking the sample and receiving the analysis result can be too long to take timely measures (i.e. change the oil). Nevertheless, other CM techniques (e.g. vibration monitoring) could still provide a way to timely predict a bearing failure, so $c 1$ is not considered to be a showstopper for ID:1.

\subsection{Selecting suitable candidates for PdM in a canal lock}

Rijkswaterstaat (RWS) is, on behalf of the Dutch ministry of infrastructure, responsible for the design, construction, management and maintenance of the main infrastructure facilities (such as highways and waterways) in the Netherlands. In this context, RWS, is responsible for numerous canal locks and pumping stations in waterways. The studied complex consists of a lock gate and a water pumping station (Figure 3). The two main functions of this complex are: controlling the water level in the waterway, and providing passage of shipping. The complex should therefore be able to pump water (increase upstream level), drain water 
(decrease upstream level), and stop the water. The moveable miter gates of the canal lock allow for the passage of shipping.

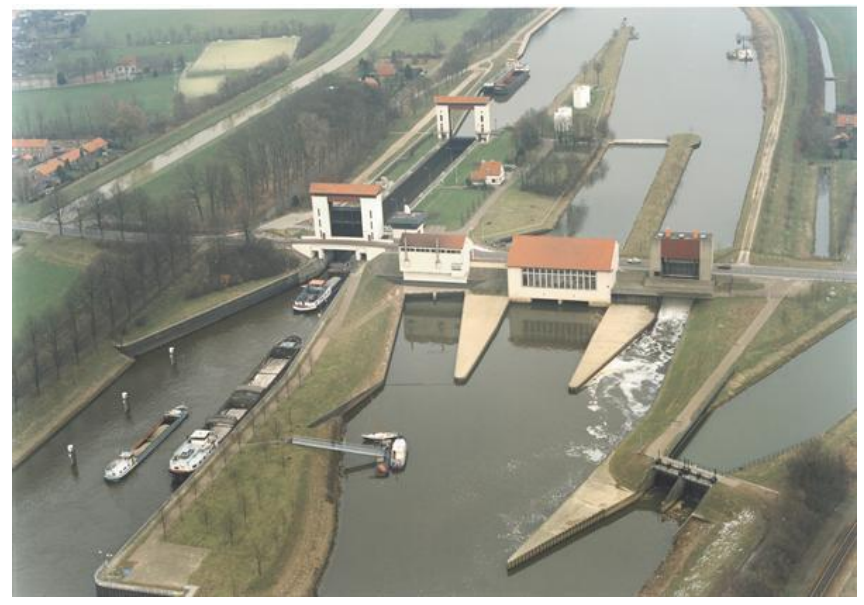

Figure 3. The canal lock (left hand side) and the water pumping station, source: RWS

RWS is currently investing in data extraction technologies that can enable PdM for the studied complex in the eastern part of the Netherlands. In this project, real time data on the operation and performance of the whole complex will become available remotely. Having the opportunity to remotely monitor the performance of e.g. the pumps in the pumping station could enable PdM. However, as many data from various installations will become available, the first question of RWS is: For which components should a predictive policy be developed first?

\subsubsection{Criticality classification}

For the first stage filtering process, existing data from a fault tree analysis (FTA) is used as input for the four quadrant chart. These FTA's were conducted by an external bureau using expert knowledge. The 175 identified events were plotted in the four quadrant chart, see Figure 4. The FTA identified 84 basic events that lead to downtime less than 24 hours (48\%), 4 events leading to downtime between a day and a week (2\%), 44 events leading to downtime between a week and a month (25\%) and 43 events leading to downtime longer than one month (25\%).

After identification of the failure modes that can be plotted in the four quadrant chart, it is important to set the boundaries for the quadrants and to set a lower level for the failure frequency, as is suggested by Tinga et al. (2017). The lower limit was set at a failure frequency of one failure every billion hours, the upper level at one failure per million hours. The lower boundary for the associated downtime was set at 24 hours (1 day).

Table 6 shows the events that were identified by applying the four quadrant chart method. 29 failures potentially interesting for PdM were identified, as they were located in Q4 of the graph. For clarity reasons, this list was cleaned up and reduced to 14 failures.

For example, similar failures for different gearboxes were grouped and also natural events influencing various installations were clustered (i.e. lightning strike for the control building and lightning strike for the tower were clustered into "lightning strike").

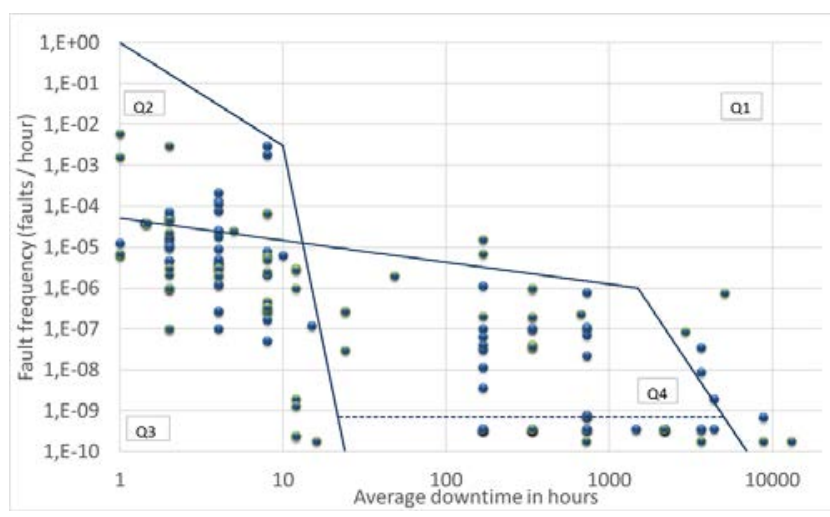

Figure 4. Four quadrant chart for the canal lock . The Q4 area indicates the failures that are suitable for PdM

\begin{tabular}{|l|l|}
\hline ID & Description of event \\
\hline 1 & Lightning strike \\
\hline 2 & Fire \\
\hline 3 & Ship collision \\
\hline 4 & Gate closed too early \\
\hline 5 & Drive hydro motor lock failure \\
\hline 6 & Cylinder leveling slider front lock failure \\
\hline 7 & Reduction gearbox failure \\
\hline 8 & Control panel pump system failure \\
\hline 9 & Return valve does not open \\
\hline 10 & Butterfly valve (electric drive) failure \\
\hline 11 & Hoist and electromotor protective gate failure \\
\hline 12 & Pump failure \\
\hline 13 & Fiber network ring connection breaks \\
\hline 14 & E-motor failure \\
\hline
\end{tabular}

Table 6. Identified candidates using the four quadrant chart method

\subsubsection{Filtering showstoppers related to the desired outcome}

The ambtion level of RWS is to be able to reduce unplanned downtime by looking at current and short-term future behavior, thus: Diagnosis. The focus of this project is to use the data that is available using the new data extraction technologies that RWS has invested in. Therefore, extra data collection is not desirable and mainly data on e-motors and pumps will become available $(t 1 a, t 2 a)$. Clustering of maintenance tasks $(c 1, c 2)$ does not give any restrictions here. 
The results of the showstopper identification show that for 8 of the 14 candidates one or more showstoppers are identified, in 4 cases (where the total result is $\mathrm{M}$ ), a more detailed feasibility study has to be conducted, and for 2 cases no showstoppers are identified at all. Oftentimes, a 'Maybe' is given for the categories 01 (trust in the monitoring system) and $o 2$ (fit to personnel). RWS is convinced that PdM has many benefits that can be gained on the long-term, but on the short-term, hick-ups can occur within the organization. Trust in the monitoring systems should be gained over time, since there is no wide spread previous experience within the organization. Next, in recent years a lot of RWS's maintenance has been outsourced. It is therefore important to consider whether sufficient domain knowledge (02: fit to personnel) is still present. RWS's goal is to build this up by investing in the conduction of failure analyses and implementing PdM. To succeed in the development of PdM for the canal lock, sufficient domain knowledge will be gained by cooperating with knowledge institutes and partners. Therefore, also RWS's relations and policies will be challenged by adopting PdM (o4). As this is labelled an 'innovation project', no insurmountable problems are foreseen. Sufficient resources (both human and financial $e 1, e 2)$ are available to solve difficulties that might occur.

\begin{tabular}{|c|c|c|c|c|c|c|c|c|c|c|c|c|}
\hline ID & $\tau$ & ช & $\stackrel{\pi}{=}$ & บี & $\vec{\jmath}$ & ช & $\overrightarrow{0}$ & ชิ & ช & $\Xi$ & 농 & 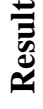 \\
\hline 1 & $\mathrm{~N}$ & $N$ & $\mathrm{Y}$ & $\mathrm{Y}$ & $\mathrm{Y}$ & $\mathrm{Y}$ & $\mathrm{Y}$ & $\mathrm{Y}$ & $\mathrm{N}$ & $N$ & $\mathrm{Y}$ & $\mathrm{Y}$ \\
\hline 2 & $\mathrm{~N}$ & $N$ & $\mathrm{Y}$ & $\mathrm{Y}$ & $\mathrm{Y}$ & $\mathrm{Y}$ & $\mathrm{Y}$ & $\mathrm{Y}$ & $\mathrm{N}$ & $\mathrm{N}$ & $\mathrm{Y}$ & $\mathrm{Y}$ \\
\hline 3 & $N$ & $N$ & $\mathrm{Y}$ & $\mathrm{Y}$ & $\mathrm{Y}$ & $\mathrm{Y}$ & $\mathrm{Y}$ & $\mathrm{Y}$ & $N$ & $N$ & $\mathrm{Y}$ & $\mathrm{Y}$ \\
\hline 4 & $N$ & $N$ & $\mathrm{Y}$ & $\mathrm{Y}$ & $\mathrm{Y}$ & $\mathrm{Y}$ & $\mathrm{Y}$ & $\mathrm{Y}$ & $N$ & $N$ & $\mathrm{Y}$ & $\mathrm{Y}$ \\
\hline 5 & $\mathrm{~N}$ & $N$ & $N$ & $N$ & $N$ & $N$ & $N$ & $N$ & $N$ & N & $N$ & $N$ \\
\hline 6 & $N$ & $N$ & $\mathrm{M}$ & $N$ & $N$ & $N$ & $\mathrm{M}$ & $\mathrm{M}$ & 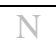 & $N$ & $N$ & $\bar{M}$ \\
\hline 7 & $\mathrm{~N}$ & $N$ & $\mathrm{M}$ & $\mathrm{N}$ & $\mathrm{M}$ & $\mathrm{N}$ & $\mathrm{M}$ & $\mathrm{M}$ & $\mathrm{N}$ & $N$ & $N$ & $\mathrm{M}$ \\
\hline 8 & $\mathrm{~N}$ & $N$ & $\mathrm{Y}$ & $\mathrm{N}$ & $\mathrm{M}$ & $\mathrm{N}$ & $\mathrm{M}$ & $\mathrm{M}$ & $\mathrm{N}$ & $\mathrm{N}$ & $\mathrm{Y}$ & $\mathrm{Y}$ \\
\hline 9 & $N$ & $N$ & $\mathrm{Y}$ & $N$ & $M$ & $N$ & $\mathrm{M}$ & $\mathrm{M}$ & $N$ & $N$ & $N$ & $\mathrm{Y}$ \\
\hline 10 & $\mathrm{~N}$ & N & $\mathrm{Y}$ & $\mathrm{N}$ & M & $N$ & $\mathrm{M}$ & $\mathrm{M}$ & $N$ & N & $N$ & $\mathrm{Y}$ \\
\hline 11 & $\mathrm{~N}$ & $N$ & $\mathrm{~N}$ & $N$ & $N$ & $N$ & $\mathrm{M}$ & $\mathrm{M}$ & $\mathrm{N}$ & $N$ & $N$ & $\mathrm{M}$ \\
\hline 12 & $N$ & $N$ & $N$ & $N$ & $N$ & $N$ & $\mathrm{~N}$ & $N$ & $N$ & $N$ & $N$ & $\mathrm{M}$ \\
\hline 13 & $\mathrm{~N}$ & $N$ & $\mathrm{Y}$ & $\mathrm{N}$ & $N$ & $N$ & $\mathrm{Y}$ & $\mathrm{M}$ & $N$ & $N$ & $\mathrm{Y}$ & $\mathrm{Y}$ \\
\hline 14 & $\mathrm{~N}$ & N & $\mathrm{N}$ & $\mathrm{N}$ & $N$ & N & $\mathrm{N}$ & $N$ & $N$ & $N$ & $N$ & $N$ \\
\hline
\end{tabular}

Table 7. Identification of showstoppers for the canal lock. $\mathrm{Y}=$ yes, $\mathrm{N}=$ no, $\mathrm{M}=$ maybe.

\subsubsection{Focused feasibility study}

The reduction gearbox (ID: 7) is one of the four failures selected as a potentially interesting candidate for PdM for which a more detailed feasibility study has to be conducted. The economic feasibility of developing PdM for the gearbox is assessed using the maintenance balanced scorecard approach, for which the results are shown in Table 8:

(i) innovation and growth: an innovative maintenance policy will be developed that helps developing competences, skills and knowledge within RWS. The impact is therefore rated considerably higher (value 5 in Table 8) compared to the traditional corrective maintenance (CM) and preventive maintenance (PM).

(ii) maintenance: The PdM introduction can reduce the nonutilized remaining life of the gearbox while complying to safety regulations and standard. However, it is not as easily plannable as fixed-interval PM activities. PdM can also decrease the work load of the maintenance organization by increasing the time between overhauls. PM and PdM are therefore scored equally. UM scores low because it can create high variances in the maintenance organization's work load and unplanned failures can occur.

(iii) production: PM and PdM can be scored equally as they both assure reliable operation and thereby guarantee the operational effectiveness of the lock.

(iv) customer: PdM might improve the availability of the lock. PdM is therefore rated slightly higher than PM, which also ensures reliable operation of the lock (the main concern of the customers).

(v) society: RWS serves the interests of society. Therefore, a reduction in the operating costs and improvements in the lock's availability are of interest to the society.

(vi) financial perspective: PdM can help to prevent costs associated with unplanned failures and damages and helps to prevent spoiling remaining useful life by only conducting maintenance when required. A downside of the introduction of PdM is the capital investment that is required for the development.

\begin{tabular}{lccc}
\hline Perspective & CM & PM & PdM \\
\hline (i) innovation and growth & 1 & 2 & 5 \\
\hline (ii) maintenance & 1 & 4 & 4 \\
\hline (iii) production & 1 & 3 & 4 \\
\hline (iv) customer & 1 & 3 & 4 \\
\hline (v) society & 1 & 3 & 4 \\
\hline (vi) financial & 1 & 3 & 4 \\
\hline Total & $\mathbf{6}$ & $\mathbf{1 8}$ & $\mathbf{2 5}$ \\
\hline
\end{tabular}

Table 8. Results of the economic feasibility study for the gearbox

The technical feasibility of developing PdM is assessed using the seven levels of the OSA-CBM structure:

1. Data acquisition: A SCADA system is available for automatic collection of sensor data (i.e. oil level, oil fouling, temperatures and motor current) and event data. The newly available data extraction technologies ensure secure data connection and back-up possibilities. The raw SCADA data is coded and send to a data center in real-time. Via this connection, asset managers are able to process the data.

2. Data processing: The project initiated by RWS focuses on developing models to process this data. Praveenkumar, Saimurugan, and Ramachandran (2017) recently showed the 
potential of using motor current signal for gearbox fault detection.

3./4. Detection and Diagnosis: Kar and Mohanty (2006) showed how motor current signal analysis can be used for gearbox fault detection. Based on the principles explained in this work, alerts can be generated based on the gearbox performance and a short-term failure prediction can be developed.

5. Prognosis: not required for the set ambition level.

6./7. Decision analysis and Presentation: via the data center, the results from the data processing can be converted to information. Dashboards and status reports are to be developed to present anomalies in behavior of the gearbox to asset managers.

In conclusion, developing a PdM approach for the gearbox seems feasible from both a strategic (financial) as well as a technical point of view.

\subsubsection{Discussion on RWS case}

Filtering showstoppers for the canal lock was helpful. After applying the four quadrant chart method, many natural events (such as lightning strikes) and external events (such as a transport accident) came up as possibly suitable candidates for PdM. Although it might be straightforward to not develop PdM for these candidates, during the session it was indicated that normally the non-technical factors that could be potential showstoppers, could easily have been ignored. The main value for RWS was therefore having a structured way of assessing the applicability of PdM per candidate. Also the feasibility study provides, at an early stage, a clear indication of how PdM can be developed using currently available technology. It points at academic literature that shows that achieving the set ambition 'Diagnosis' with the existing sensors is indeed possible.

\section{CONCLUSION}

This paper aimed to propose a method to select the suitable components for PdM / PHM within an asset. It can be concluded that the proposed three stage approach can be widely applied for suitable candidate selection, as was demonstrated by the canal lock (RWS) and naval vessel propulsion system (MAR) cases. Applying the four quadrant chart as a first filter has shown to reduce the time effort as it significantly reduces the number of potential candidates with up to $90 \%$ for MAR and $84 \%$ for RWS. The second stage, i.e. identifying showstoppers proves to be an important contribution of this paper. In both cases, several showstoppers have been identified that are easily overlooked by traditional methods (as the cylinder liner example in the MAR case clearly showed). Thus, simply applying PdM / PHM on the top cost drivers or performance killers will in many cases not lead to optimal results (i.e. reducing downtime or maintenance costs by applying PdM). Besides, the value of the proposed method is not only in generating a list of suitable candidates for PdM, but also in providing a structured and traceable way to determine these candidates. By identifying potential showstoppers in advance and conducting a structured feasibility study, the often observed trial-and-error approach in developing PdM in practice can be prevented.

The cases also show that the group of persons applying the proposed method affect the results, since especially the showstopper identification and the feasibility study are fairly subjective. This is however not problematic by itself: although attention is required to create reproducible results, the goal of the method is to assess showstoppers and the feasibility within the company. It is nevertheless advised to use a multidisciplinary team which is well suited to estimate the feasibility within the firm. This feasibility is determined by e.g. the knowledge level of employees, maturity of the firm, previous experiences with PdM and experience in applying the proposed selection method.

Finally, the proposed method has only be tested in situations where a clear project aiming at developing PdM was initiated. This might also explain why only a limited number of organizational showstoppers have been identified; the companies were ready to start with implementing PdM. Besides, the proposed list of showstoppers might not be complete for any application, but can easily be extended with additional factors when necessary. Further research should therefore focus on testing the proposed method in standard maintenance improvement programs in practice.

\section{ACKNOWLEDGEMENT}

The authors wish to express gratitude to the interviewees for their contributions to this paper. This research is part of the Tools4LCM project, funded by the Netherlands Ministry of Defence and the National Aerospace Centre NLR. The research is also part of the MaSeLMA project (Integrated Maintenance and Service Logistics Concepts for Maritime Assets), funded by Dinalog (Dutch Institute for Advanced Logistics).

\section{REFERENCES}

Alsyouf, I. (2006). Measuring maintenance performance using a balanced scorecard approach. Journal of Quality in Maintenance Engineering, 12(2), 133149. doi:10.1108/13552510610667165

Avizienis, A., Laprie, J.-C., Randell, B., \& Landwehr, C. (2004). Basic concepts and taxonomy of dependable and secure computing. IEEE transactions on dependable and secure computing, 1(1), 11-33.

Banks, J. C., Reichard, K. M., Hines, J. A., \& Brought, M. S. (2008). Platform degrader analysis for the design and development of vehicle health management systems. Paper presented at the International 
Conference on Prognostics and Health Management.

Bengtsson, M. (2008). A Method for Implementing Condition Based Maintenance in Industrial Settings. Paper presented at the 18th international conference on flexible automation and intelligent manufacturing, Skövde.

Bengtsson, M., \& Jackson, M. (2004). Important aspects to take into consideration when deciding to implement condition based maintenance. Paper presented at the 17th International Conference on Condition Monitoring and Diagnostic Engineering Management.

Brahimi, M., Medjaher, K., Leouatni, M., \& Zerhouni, N. (2016). Critical Components Selection for a Prognostics and Health Management System Design: an Application to an Overhead Contact System. Paper presented at the Annual conference of the prognostics and health management society Denver, Colorado.

Dehghanian, P., Fotuhi-Firuzabad, M., Bagheri-Shouraki, S., \& Kazemi, A. A. R. (2012). Critical component identification in reliability centered asset management of power distribution systems via fuzzy AHP. IEEE Systems Journal, 6(4), 593-602.

Duplex, P. (2017). Predictive maintenance concepts for maritime applications: PdEng qualifier report. University of Twente. Enschede, Netherlands.

Goossens, A. J., \& Basten, R. J. (2015). Exploring maintenance policy selection using the Analytic Hierarchy Process; an application for naval ships. Reliability Engineering \& System Safety, 142, 3141.

Gouriveau, R., Medjaher, K., \& Zerhouni, N. (2016). From Prognostics and Health Systems Management to Predictive Maintenance 1: Monitoring and Prognostics: John Wiley \& Sons.

Hashemian, H. M., \& Bean, W. C. (2011). State-of-the-art predictive maintenance techniques. IEEE Transactions on Instrumentation and measurement, 60(10), 3480-3492.

Holmström, J., Ketokivi, M., \& Hameri, A. P. (2009). Bridging practice and theory: a design science approach. Decision Sciences, 40(1), 65-87.

Jardine, A. K. S., Lin, D., \& Banjevic, D. (2006). A review on machinery diagnostics and prognostics implementing condition-based maintenance. Mechanical Systems and Signal Processing, 20(7), 1483-1510. doi:http://dx.doi.org/10.1016/j.ymssp.2005.09.012

Jonsson, K., \& Westergren, U. H. (2004). Developing remote monitoring services: Important points to consider. Paper presented at the 27th Information systems research seminar in scandinavia (Iris 27).

Kar, C., \& Mohanty, A. R. (2006). Monitoring gear vibrations through motor current signature analysis and wavelet transform. Mechanical Systems and Signal Processing, 20(1), 158-187. doi:https://doi.org/10.1016/j.ymssp.2004.07.006

Labib, A. W. (2004). A decision analysis model for maintenance policy selection using a CMMS. Journal of Quality in Maintenance Engineering, 10(3), 191-202.

LaRiviere, J., McAfee, P., Rao, J., Narayanan, V. K., \& Sun, W. (2016, 25 may 2016). Where Predictive Analytics Is Having the Biggest Impact. Retrieved from https://hbr.org/2016/05/where-predictiveanalytics-is-having-the-biggest-impact

Lebold, M., Reichard, K., \& Boylan, D. (2003, March 8-15, 2003). Utilizing dcom in an open system architecture framework for machinery monitoring and diagnostics. Paper presented at the Aerospace Conference, 2003. Proceedings. 2003 IEEE.

Lee, J., Liao, L., Lapira, E., Ni, J., \& Li, L. (2009). Informatics platform for designing and deploying emanufacturing systems. Collaborative Design and Planning for Digital Manufacturing, 1-35.

Lee, J., Wu, F., Zhao, W., Ghaffari, M., Liao, L., \& Siegel, D. (2014). Prognostics and health management design for rotary machinery systems-Reviews, methodology and applications. Mechanical Systems and Signal Processing, 42(1), 314-334.

Lei, X., \& Sandborn, P. A. (2016). PHM-based wind turbine maintenance optimization using real options. Int $J$ Progn Health Manag, 7(1), 1-14.

Lewis, R., Dwyer-Joyce, R., Slatter, T., \& Brooks, A. (2004). Valve recession: From experiment to predictive model. VDI Berichte(1813), 79-93.

Moubray, J. (1997). RCM II, Reliability-centred maintenance. New York: Industrial Press Inc.

Peeters, J. F. W., Basten, R. J. I., \& Tinga, T. (2018). Improving failure analysis efficiency by combining FTA and FMEA in a recursive manner. Reliability Engineering \& System Safety, 172, 36-44. doi:https://doi.org/10.1016/j.ress.2017.11.024

Pintelon, L., \& Van Puyvelde, F. (2006). Maintenance decision making. Leuven, Belgium Acco.

Praveenkumar, T., Saimurugan, M., \& Ramachandran, K. I. (2017). Comparison of vibration, sound and motor current signature analysis for detection of gear box faults. International Journal of Prognostics and Health Management, 8(2).

Shafiee, M. (2015). Maintenance strategy selection problem: an MCDM overview. Journal of Quality in Maintenance Engineering, 21(4), 378-402.

Simões, J. M., Gomes, C. F., \& Yasin, M. M. (2016). Changing role of maintenance in business organisations: measurement versus strategic orientation. International journal of production research, 54(11), 3329-3346. doi:10.1080/00207543.2015.1106611 
Tiddens, W. W., Braaksma, A. J. J., \& Tinga, T. (2017). Towards Informed Maintenance Decision Making: Guiding the Application of Advanced Maintenance Analyses. In M. C. Carnero \& V. González-Prida (Eds.), Optimum Decision Making in Asset Management (pp. 288-309). Hershey, PA: IGI Global.

Tiddens, W. W., Brouwer, O., Braaksma, A. J. J., \& Tinga, T. (2017). The business case for condition-based maintenance: a hybrid (non-) financial approach Paper presented at the Safety and Reliability Theory and Applications, Portoroz, Slovenia.

Tinga, T., \& Loendersloot, R. (2014). Aligning PHM, SHM and $C B M$ by understanding the system failure behaviour. Paper presented at the European Conference of the prognostics and health management society 2014, Nantes.

Tinga, T., Tiddens, W. W., Amoiralis, F., \& Politis, M. (2017). Predictive maintenance of maritime systems. Paper presented at the 27th European Safety and Reliability Conference (ESREL 2017).

Tsang, A. H. (1995). Condition-based maintenance: tools and decision making. Journal of Quality in Maintenance Engineering, 1(3), 3-17.

Waeyenbergh, G., \& Pintelon, L. (2002). A framework for maintenance concept development. International Journal of Production Economics, 77(3), 299-313.

\section{BIOGRAPHIES}

Wieger Tiddens is a PhD candidate at the University of Twente and the Netherlands Defence Academy. He holds a Master's degree in Technology Management obtained at the University of Groningen. He has professional experience in the application of maintenance concepts and analytics within industry. His research interests include the support of informed maintenance decision making and the application of predictive maintenance within companies.

Jan Braaksma is an assistant professor in the chair of Maintenance Engineering and director of the WCM Summer School. He is a member of the maintenance research center TIME. He has worked for the University of Groningen (RuG) and the Netherlands Defense Academy (NLDA). He holds a Master's degree in Business and ICT and a PhD degree in Economics and Business and conducts research on Maintenance Engineering, Asset information and life-cycle management.

Tiedo Tinga is a full professor in dynamics based maintenance at the University of Twente since 2012 and full professor Life Cycle Management at the Netherlands Defence Academy since 2016. He received his $\mathrm{PhD}$ degree in mechanics of materials from Eindhoven University in 2009. He is chairing the maintenance research center TIME and now leads a number of research projects on developing predictive maintenance concepts, mainly based on physics of failure models, but also following data-driven approaches. 